\title{
Pfaffian States in Coupled Atom-Cavity Systems
}

\author{
Andrew L.C. Hayward and Andrew M. Martin \\ School of Physics, University of Melbourne, Vic 3050, Australia
}

(Dated: June 18, 2021)

\begin{abstract}
Coupled atom-cavity arrays, such as those described by the Jaynes-Cummings Hubbard model, have the potential to emulate a wide range of condensed matter phenomena. In particular, the strongly correlated states of the fractional quantum Hall effect can be realised. At some filling fractions, the fraction quantum Hall effect has been shown to possess ground states with nonabelian excitations. The most well studied of these states is the Pfaffian state of Moore and Read, which is the groundstate of a Hall Liquid with a 3-body interaction. In this paper we show how an effective 3-body interaction can be generated within the Cavity QED framework, and that a Pfaffian-like groundstate of these systems exists.
\end{abstract}

PACS numbers: 42.50.pq, 73.43.-f, 32.80.Qk

Coupled atom-cavity systems (falling under the broad umbrella of Cavity QED) is proving to be an excellent framework for the investigation of fundamental quantum phenomena, due to the versatility and control of system parameters. In particular, cavity QED is promising to be a powerful platform for quantum emulation, where a wide range of condensed matter systems can be modeled through tailoring of atom-cavity interactions 1 [ 6 .

Recent work has shown how complex states of correlated light can be induced in cavity QED systems via non-linearities in atom-light interactions. In particular, a class of states that correspond to the quantum Hall effect have been predicted. In this paper we extend this class to include Pfaffian-like states [7]. These states possess highly non-trivial topological properties, that make them of great interest to the understanding of quantum Hall physics, as well as providing an opportunity to examine strongly correlated quantum states of light.

Since the discovery of fractional quantum Hall states [8], a small industry has grown up around the construction of complex states that might be found in 2dimensional quantum Hall fluids. These states (for the most part) lie in the massively degenerate lowest Landau level (LLL). Single particle states in the lowest Landau level have a beautiful property that leads to the general structure found across all the QHE states. One can write the position of a single particle $k$ in the 2D plane as $z_{k}=\left(x_{k}+i y_{k}\right) / \ell_{B}$, with $\ell_{B}$ the magnetic length, then the single particle states can all be written as a holomorphic function in $z_{k}$, multiplied by a Gaussian factor. As a consequence, all many-body states that lie in the LLL are functions of $\bar{z}=\left\{z_{k} \mid k \leq N_{p}\right\}$, and consist of a Gaussian term, $\exp \left[-\frac{1}{4} \sum_{k}\left|z_{k}\right|^{2}\right]$, multiplied by a Jastrow Factor, an analytic function $F(\bar{z})$.

The Jastrow factor encodes the correlations between particles. The simplest correlated state was proposed by Laughlin [9] to account for the fractional QHE states found at $\nu=1 / q$. The Laughlin Jastrow factors are $F_{L}(\bar{z})=\prod_{k>j}\left(z_{k}-z_{j}\right)^{q}$. These states have a wavefunction that vanishes as any two particles apporach one an- other, minimizing the electron-electron repulsion within the lowest Landau level.

The Moore-Read Pfaffian state is defined by the Jastrow factor

$$
\Psi^{\mathrm{Pf}} \propto \operatorname{Pf}\left(\frac{1}{z_{k}-z_{j}}\right) \prod_{k>j}\left(z_{k}-z_{j}\right)^{q} .
$$

The terms in the Pfaffain factor cancel a factor in the Laughlin state, leading to a non-zero amplitude at the coincidence of just two particles.

This ground state has some interesting properties; most notably, non-abelian excitations, and (relatedly) a triply degenerate groundstate in the toroidal geometry [7].

In this work we consider a Jaynes-Cummings-Hubbard ( $\mathrm{JCH})$ system with synthetic magnetic field and demonstrate the existence of Pfaffian-like groundstates at a filling factor $\nu=1$. We first consider the conventional $\mathrm{JCH}$ system, and find no evidence for Pfaffian-like states. We then consider a three level Jaynes-Cummings (JC) cavity and show how there are regimes in which 3-body interactions dominate. We then study the conditions under which Pfaffian-like states will arise when these 3 level systems are Hubbard-coupled.

Each cavity in the JCH lattice is described by:

$$
H^{J C}=\omega L+\Delta \sigma^{+} \sigma^{-}+\beta\left(\sigma^{+} a+\sigma^{-} a^{\dagger}\right),
$$

where $a$ is the photonic annihilation operator, $\sigma^{ \pm}$are the atomic raising and lowering operators, $L$ is the excitation number operator, $\Delta$ the atom-photon detuning, $\beta$ the coupling energy and $\hbar=1$. The states $|g(e), n\rangle$, where $n$ is the number of photons, and $g(e)$ are the ground (excited) state of the atom, form the single cavity basis. $H^{J C}$ commutes with the total excitation number operator, $L$. Therefore the total excitations in the cavity, $\ell$, is a good quantum number. The eigenstates of Eq. (2) are termed polaritons, superpositions of atomic and photonic excitations, and are a function of $\ell$ and $\Delta / \beta$.

The JCH model describes an array of individual Jaynes-Cummings cavities, which are coupled via a Hub- 
bard like photon tunneling term. In the case of a lossless system, the JCH model can be described by:

$$
H^{J C H}=\sum_{i} H_{i}^{J C}-\sum_{\langle i, j\rangle} \kappa_{i j} a_{i}^{\dagger} a_{j}
$$

where $\kappa_{i j}$ is the tunneling rate between cavities $i$ and $j$ and the sum over $\langle i, j\rangle$ is between nearest neighbors only.

For large detuning $(|\Delta| \gg \beta)$, eigenstates separate out into either atomic or photonic modes. In this limit, the photonic or atomic mode can be adiabatically eliminated. Eliminating the atomic modes, the photonic mode has a weak Kerr-type photon-photon repulsion [10] and the exchange of energy between atomic and photonic modes is strongly suppressed. However, virtual processes lead to effective interactions in the photonic and atomic submanifolds. Photons have an atomic mediated non-linear onsite repulsion, making the JCH model equivalent to the Bose-Hubbard (BH) model [1]. Atomic modes are coupled with the effective hopping rate $\kappa_{i j}^{e f f}=\kappa_{i j} \beta^{2} / \Delta^{2}[\underline{12}$. As the atomic modes are restricted to two levels, this is effectively a hardcore boson field for atomic states, in contrast to the weakly-interacting photon field.

Investigation of quantum Hall physics in the $\mathrm{JCH}$ model requires the introducion of a synthetic magnetic field. An artificial magnetic field may be realized via the introduction of some time reversal symmetry breaking interaction. A number of techniques have been proposed to achieve this [6, 13 17]. For example, one may exploit a time-dependent potential to induce magnetic flux across the lattice [16], thereby explicitly breaking the time symmetry of the system. A similar strategy is proposed in Ref. [6], where the authors utilize a time dependent intersite coupling to induce a synthetic magnetic field. Alternatively, effective coupling to real magnetic fields can be used, such as in the proposal by Koch et al. [15]. Other means, such as the use of optically polarized media [13] or via atomically mediated inter-site coupling 14 have also been proposed.

Inspired by results in ultra cold atom simulations 18 21], one might expect to find evidence of a Pfaffian groundstate in the JCH model at $\nu=1$. We numerically investigated the $\mathrm{JCH}$ model on a torus to this end. Existence of a Pfaffian like state can be indicated by a number of properties of the groundstate: a triply degenerate groundstate manifold, large overlap with the Pfaffian trial wavefunction, and a Chern number of $3 \mathrm{com}-$ puted for the three groundstates.

We conducted a comprehensive search over several parameters within the JCH model, but the tell-tail signature of a triple degeneracy groundstate proved elusive. Instead, simulations reveal that for some lattice configurations a single separated groundstate in the strongly interacting limit, with a transition to a gapless phase as the effective 2-body interaction decreased. Other configurations possessed a gapless groundstate extending all the way to the hardcore limit. While these results jar with the Bose-Einstein condensate (BEC) findings, other lattice boson simulations have failed similarly 22.

Although the evidence from BECs suggests that a Pfaffian groundstate should be preferred, there are other possible states at $\nu=1$ filling for Bosons which are in competition with the Pfaffian state. For example, Read 23] proposes a groundstate in which, approximately, a single vortex is attached to each Boson. This assignment exactly cancels out the external magnetic field, which reduces the problem to that of a Fermi-Liquid. Alternatively, it is conjectured 24] that a striped phase with charge density order may exist at $\nu=1$, with some numerical simulations 25] finding evidence for this.

The relativly small size of the systems we have simulated make it difficult to tease out the importance of different effects which determine the real nature of the groundstate at this filling factor. However, the poor scaling of these systems means that significantly larger systems are impractical at this point in time. Of course, this problem is one of the primary motivators of work into quantum emulation.

For $\nu=1$, the Pfaffian groundstate is the highest density groundstate of the 3-body delta potential Hamiltonian [26]. For each pair of particles, there is a number of terms in Eq. (11) for which each particle is in a different partition. However, there is no term for which 3 or more particles coincide that does not vanish.

The JCH in the limit of large detuning can be described by a Bose-Hubbard with an effective two body interaction, $U_{2}$. However, the atomic-cavity interaction induces interactions to all orders of n-photon interactions. These higher order interactions are much smaller than the two body interaction, and therefore the physics of the $\mathrm{JCH}$ very much mirrors that of the Bose-Hubbard model with two body interaction. We now show that it is possible to eliminate the two-body interaction while retaining the higher order interactions.

Three-body interactions (without corresponding 2body ones) are unnatural, and do not arise in many physical systems. A number of schemes for creating effective 3 -body interactions have been proposed in the context of BECs 22, 27 29] and in the circuit QED setting [30]. Below we show that an effective 3-body interaction can be induced in atom-cavity lattice by replacing the 2-level atoms in the JCH model with appropriately tuned 3level atoms. Furthermore, it is demonstrated via simulation that an atom-cavity lattice consisting of these 3-level atoms can possess a Pfaffian like state as its groundstate.

We consider the 3 level system atom in the $\Xi$ configuration, as shown in Fig. 1 1 a). This configuration consists of two evenly spaced excited states, with the atom cavity system described by the Hamiltonian:

$$
\begin{aligned}
H^{3 L}=\omega a^{\dagger} a & +\epsilon_{1}\left|e_{1}\right\rangle\left\langle e_{1}\right|+\left(\beta_{1} \sigma_{1}^{+} a+\text { h.c. }\right) \\
& +\epsilon_{1}\left|e_{2}\right\rangle\left\langle e_{2}\right|+\left(\beta_{2} \sigma_{2}^{+} a+\text { h.c. }\right) .
\end{aligned}
$$


a)

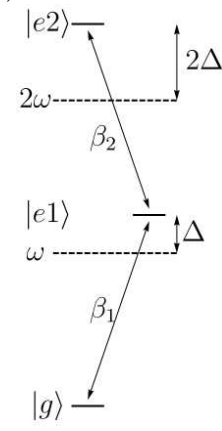

b)

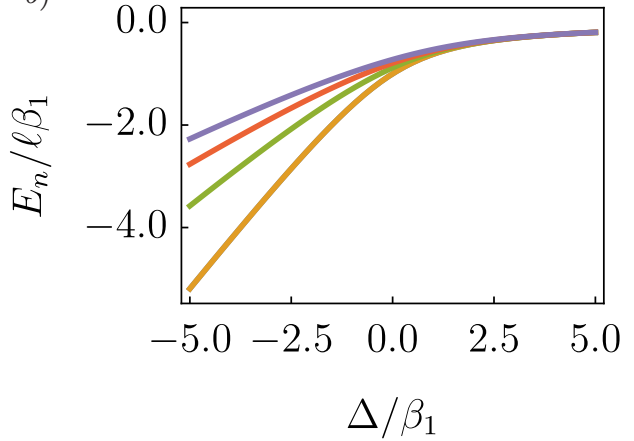

c)

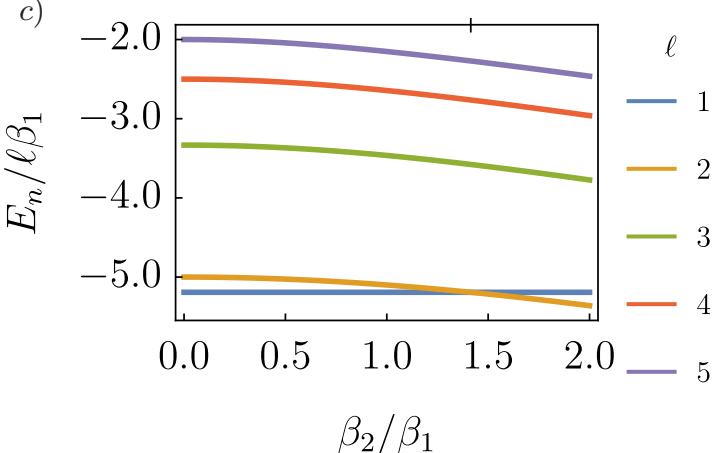

FIG. 1. a) 3 level atom configuration for inducing a 3-body interaction in the JCH lattice. b) Energy per excitation in the 3-level atom for $n=1-5$ (blue, orange, green red, purple in order). Excitations in the $n=1$ and $n=2$ excitation subspace have the same energy cost. For higher excitation numbers, the energy per particle increases. c) Energy per excitation as a function of $\beta_{2}$ (same colors as b) for $\Delta / \beta_{1}=-5$.

Here, $\sigma_{1(2)}^{+}$raises the atomic level from $g \leftrightarrow e_{1}\left(e_{1} \leftrightarrow e_{2}\right)$, and levels $1(2)$ have energies $\epsilon_{1(2)}$.

Choosing an atom with energy levels:

$$
\epsilon_{1}=\omega-\Delta, \quad \epsilon_{2}=2 \omega-2 \Delta,
$$

and transition strengths:

$$
g \leftrightarrow e_{1} \equiv \beta_{1}, \quad e_{1} \leftrightarrow e_{2} \equiv \beta_{2}=\sqrt{2} \beta_{1},
$$

leads to an effective 3-body interaction. This can be seen by considering the formulation of the JC system as a twomode Bosonic system with inter-mode tunneling. If one imposes a hardcore boson condition on one of the modes, then the system corresponds exactly to the JC cavity with a two-level atom. In the single excitation subspace, the hardcore condition is automatically satisfied, and the system is simply a free boson model.

If, instead of the hardcore condition, one imposes a 3-particle hardcore condition $\left(U_{3} \rightarrow \infty\right)$, then a similar situation arises, except that for both the single and double excitation subspaces, there are no interactions. Mapping this model back to the atomic model, for 1 and 2 particles, the equivalent atom-cavity model corresponds exactly to the one presented previously, where the factor of $\sqrt{2}$ arises from the indistinguishably of the bosons.

The 3-body non-linearity is demonstrated in Fig. 1 b). Here, the energy cost per particle for the lower polariton branch is plotted as a function of the detuning, $\Delta$. For 1 and 2 excitations, the energy per particle is the same. However, for $\ell=3$ and above, there is an increased cost for adding additional particles. Figure 15) shows that, at $\beta_{2} / \beta_{1}=\sqrt{2}$ the 2 and 3 level atom-cavity systems share several properties. The non-linearity is unbounded as the detuning is lowered, and disappears as the detuning is increased. Also, the non-linearity does not grow quadratically with excitation number, as is the case for a pure 3-body interaction, similar to the 2-level atom case.

Figure 1 ) demonstrates how the two body interaction is affected by the strength of $\beta_{2}$. As $\beta_{2}$ is tuned away from $\beta_{2} / \beta_{1}=\sqrt{2}$ the effective two-body interaction becomes
+ ve or -ve. However, at low detunings $\left(\Delta / \beta_{1}=-5\right.$ in Fig. 11) the relative strength of this effective two body interaction is much smaller than the three body one.

This method for generating 3-body interactions opens up unique possibilities for investigating the physics of topological quantum states that has proved elusive in traditional environments. Furthermore, this same technique can be extended to higher order interactions. There is a hierarchy of states that generalize the Pfaffian 31] state, which are expected to be groundstates of these higher order interactions. The JCH model with this modification is, to our knowledge, the only system in which such higher order interactions might be achieved, outside of a fully functional quantum computer.

In practice, engineering the three level system as described lies well within the capabilities of current cavity QED fabrication techniques. Engineering a system like this in circuit QED has been discussed in [30]. For cavity atoms, most $\Xi$ configurations tend to be unstable, with fast relaxation rates that would preclude large scale coherence in the system. This instability can be mitigated by instead using an $M$ like configuration (as in 32]), where classical driving can be used to create an effective three level JCH system.

In our simulations we restrict the system to a torus, to remove edge effects. The toroidal geometry permits twisted periodic boundary conditions which reduces finite size effects, and allows for computation of the Chern number.

The Chern number 33], $\mathcal{C}$, is a measure of the topology of the groundstate of the system and can provide evidence for the existence of a Pfaffian like state. Here, we define a two dimensional manifold over the two phases, $\theta_{x, y}$, that parametrize the twisted periodic boundary conditions across the torus. For the Pfaffian state, the three groundstates have a combined Chern number of 3 .

We compute, by exact diagonalization, the low energy band structure of the 3 level JCH system for 4 excitations over the twisted boundary condition manifold [Fig. 2]a)]. For 4 particles there is a large modulation of the energy 


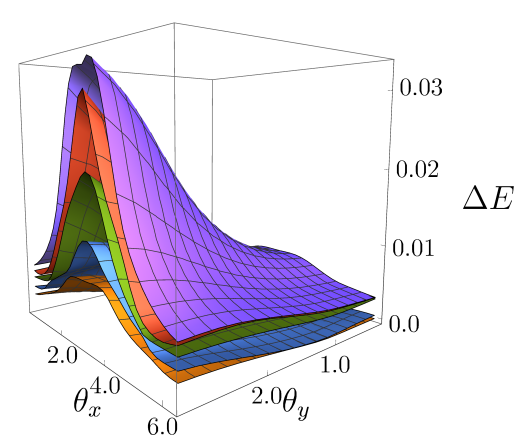

I

1

c

t

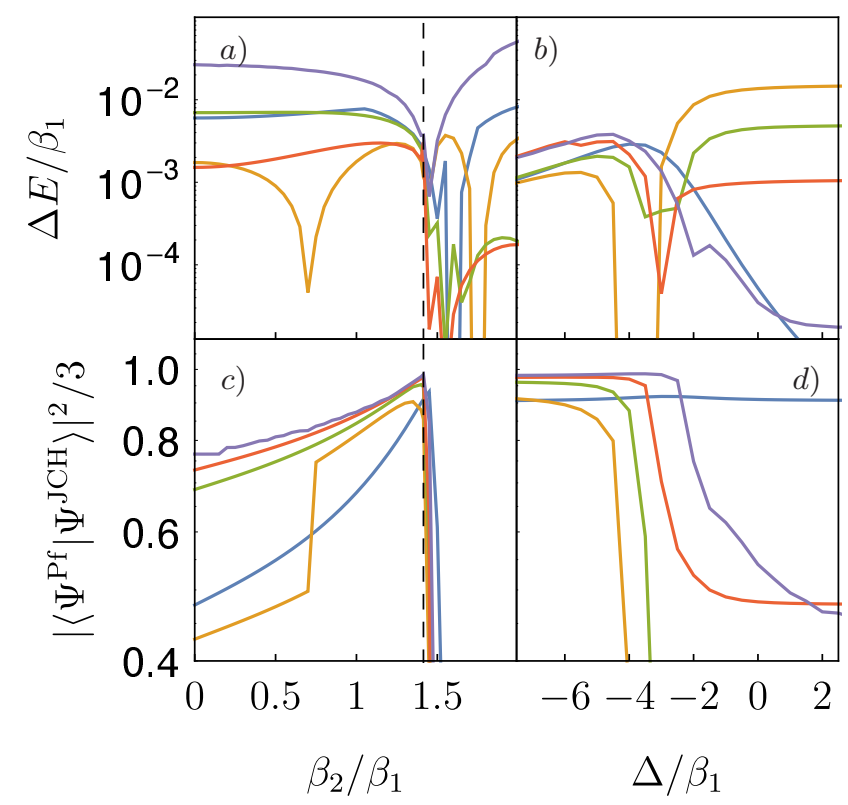

FIG. 3. Bandgap and Pfaffian overlap as a function of $\beta_{2} / \beta_{1}$ (at $\Delta / \beta_{1}=-5$ ) and $\Delta / \beta_{1}$ (with $\beta_{2} / \beta_{1}=\sqrt{2}$ ) for 4 particles on $4 \times 4$ (blue) $4 \times 5$ (orange) $5 \times 5$ (green) $6 \times 4$ (red) $6 \times 5$ (purple) and $6 \times 6$ (brown) lattice.

as a function of the twist angles (which one expects to dissapear in the many-particle limit 34]). We find that, a quasi-gap can exist in both models and computation of the Chern number $(\mathcal{C}=3)$, coupled with the 3 -fold degeneracy provides strong evidence for Pfaffian physics.

In the case of Laughlin states on a lattice, the gap has been shown [33] to scale proportianlly to the flux density per lattice plaquette. This does not seem to apply in the case of the Pfaffian state. We hypothesise that the first excited state of this system is not an excitation lying in a higher Landau level. Rather, it is some combination of quasi-particles and quasi-holes. These excitations lie in the LLL, with no energy gap in the continuum limit, which would explain the rapid decrees in the gap as the flux density per lattice plaquette decreases.
With strong evidence for the Pfaffian state, we proceed to investigate the 3-level JCH model in more detail, by exploring the properties of the groundstate over a range of lattice sizes and system parameters. These investigations are presented in Fig. 3 ,

In Figs. 3 a) and c) we show how the groundstate changes as a function of $\beta_{2}$. The groundstate experience a transition from the Pfaffian state away from $\beta_{2} / \beta_{1}=\sqrt{2}$. For $\beta_{2} / \beta_{1}>\sqrt{2}$, where the effective two body interaction becomes attractive, there is a very sharp transition to a collapsed state. On the other side, $\beta_{2} / \beta_{1}<\sqrt{2}$, the gap and Pfaffian overlap remain fairly stable, although we observe a transition in the $4 \times 5$ lattice configuration.

In Figs. 3b) and d) we show how the groundstate changes as a function of the detuning, $\Delta$. We find that, as in the case of the Laughlin case 35], increasing the atomic detuning, which alters the effective interaction strength (Fig. 1b), can induce a transition from a Pfaffian state to an uncorrelated one. This transition is accompanied by a closing of the bandgap, and, for most cases, a dropoff of the overlap with the trial wavefunction.

The Pfaffian states at $\nu=1$ have a straightforward interpretation as the symmetrised product of two Laughlin states at $\nu=1 / 2$ [36. Assigning each particle to one of two Laughlin states. The wavefunction will vanish as two particles in the same Laughlin state approach each other, but not if those two particles are in different states. However, if any three particles coincide, then by construction the wavefunction will be zero at this point. The three degenerate states in the torus setting correspond to the singlet and two doublet states one can construct from the doubly degenerate Laughlin states.

This re-expression of the Pfaffian wavefunction also allows one to translate findings from investigations into the Laughlin state in the JCH into the current work. For example, we find that the detuning for which the Pfaffian state undergoes a transition (Fig. 3 3 ,d) is the same for the equivalent single Laughlin state 35]. Furthermore, the overlap with the trial Pfaffian wavefunction is very well approximated by the overlap with a single Laughlin function, to the power of two.

In conclusion, we have described a method by which three-body interactions can be induced in JaynesCummings-Hubbard systems. In the presence of synthetic magnetic fields, such interactions, strongly correlated states of light, with Pfaffian-like topological properties, will exist. This opens up exciting possibilities for the exploration of exotic quantum states within the cavity QED framework, including states with non-abelian quasi-particles pertaining to topological quantum computing.

The authors would like to acknowledge A.D. Greentree for helpful disucssions. 
[1] A. D. Greentree, C. Tahan, J. H. Cole, and L. C. L. Hollenberg, Nature Physics 2, 856 (2006), ISSN 17452473.

[2] D. Rossini and R. Fazio, Physical Review Letters 99 (2007), ISSN 0031-9007.

[3] J. Quach, M. I. Makin, C.-H. Su, A. D. Greentree, and L. C. L. Hollenberg, Physical Review A 80 (2009), ISSN 1050-2947.

[4] D. Gerace, H. E. Türeci, A. Imamoglu, V. Giovannetti, and R. Fazio, Nature Physics 5, 281 (2009), ISSN 17452473.

[5] J. Q. Quach, C.-H. Su, A. M. Martin, A. D. Greentree, and L. C. L. Hollenberg, Optics express 19, 11018 (2011), ISSN 1094-4087.

[6] E. Kapit, M. Hafezi, and S. H. Simon, Physical Review X 4, 1 (2014), ISSN 2160-3308, arXiv:1402.6847v1.

[7] G. Moore and N. Read, Nuclear Physics B 360, 362 (1991), ISSN 05503213.

[8] D. C. Tsui, H. L. Stormer, and A. C. Gossard, Physical Review Letters 48, 1559 (1982), ISSN 0031-9007.

[9] R. B. Laughlin, Physical Review Letters 50, 1395 (1983), ISSN 0031-9007.

[10] N. Na, S. Utsunomiya, L. Tian, and Y. Yamamoto, Physical Review A 77, 031803 (2008), ISSN 1050-2947.

[11] M. Hohenadler, M. Aichhorn, S. Schmidt, and L. Pollet, Physical Review A 84 (2011), ISSN 1050-2947.

[12] M. Makin, J. Cole, C. Hill, A. Greentree, and L. Hollenberg, Physical Review A 80 (2009), ISSN 1050-2947.

[13] F. Haldane and S. Raghu, Physical Review Letters 100 (2008), ISSN 0031-9007.

[14] J. Cho, D. Angelakis, and S. Bose, Physical Review Letters 101, 4 (2008), ISSN 0031-9007, 0807.1802.

[15] J. Koch, A. Houck, K. L. Hur, and S. Girvin, Physical Review A 82 (2010), ISSN 1050-2947.

[16] A. R. Kolovsky, EPL (Europhysics Letters) 93, 20003 (2011), ISSN 0295-5075.

[17] R. O. Umucalilar and I. Carusotto (2011), 1104.4071.

[18] N. Cooper, N. Wilkin, and J. Gunn, Physical Review Letters 87, 120405 (2001), ISSN 0031-9007.
[19] N. Regnault and T. Jolicoeur, Physical Review B 69, 235309 (2004), ISSN 1098-0121.

[20] C.-C. Chang, N. Regnault, T. Jolicoeur, and J. Jain, Physical Review A 72, 013611 (2005), ISSN 1050-2947.

[21] M. Peterson, T. Jolicoeur, and S. Das Sarma, Physical Review Letters 101 (2008), ISSN 0031-9007.

[22] L. Mazza, M. Rizzi, M. Lewenstein, and J. Cirac, Physical Review A 82 (2010), ISSN 1050-2947.

[23] N. Read, Physical Review B 58, 16262 (1998), ISSN 0163-1829.

[24] E. H. Rezayi, F. D. M. Haldane, and K. Yang, Physical Review Letters 83, 1219 (1999), ISSN 0031-9007.

[25] B. Chung and T. Jolicœur, Physical Review A 77 (2008), ISSN 1050-2947.

[26] M. Greiter, X. Wen, and F. Wilczek, Nuclear Physics B 374, 567 (1992), ISSN 05503213.

[27] H. P. Büchler, A. Micheli, and P. Zoller, Nature Physics 3, 726 (2007), ISSN 1745-2473.

[28] A. Daley, J. Taylor, S. Diehl, M. Baranov, and P. Zoller, Physical Review Letters 102, 040402 (2009), ISSN 00319007.

[29] A. J. Daley and J. Simon, Physical Review A 89, 053619 (2014), ISSN 1050-2947.

[30] M. Hafezi, P. Adhikari, and J. M. Taylor, Physical Review B 90, 060503 (2014), ISSN 1098-0121.

[31] N. Read and E. Rezayi, Physical Review B 59, 8084 (1999), ISSN 0163-1829.

[32] A. D. Greentree, J. A. Vaccaro, S. R. de Echaniz, A. V. Durrant, and J. P. Marangos, Journal of Optics B: Quantum and Semiclassical Optics 2, 252 (2000), ISSN 14644266.

[33] M. Hafezi, A. Sørensen, E. Demler, and M. Lukin, Physical Review A 76, 023613 (2007), ISSN 1050-2947.

[34] R. Varnhagen, Nuclear Physics B 443, 501 (1995), ISSN 05503213.

[35] A. L. C. Hayward, A. M. Martin, and A. D. Greentree, Physical Review Letters 108, 223602 (2012), ISSN 00319007.

[36] N. Read and E. Rezayi, Physical Review B 54, 16864 (1996), ISSN 0163-1829. 